\title{
SNR Maximization using Fuzzy Rule based System in Relay Assisted Cognitive Radio Networks
}

\author{
Kiran Sultan \\ Dept. of CIT \\ King Abdul Aziz \\ University \\ Jeddah, Saudi Arabia
}

\author{
Bassam A. Zafar \\ Dept. of CIT \\ King Abdul Aziz University \\ Jeddah, Saudi Arabia
}

\author{
Waseem Khan \\ Dept. of EE \\ National University of \\ Comp. and Emerging Sci. \\ Islamabad, Pakistan
}

\author{
Atta-Ur-Rahman \\ Barani Institute of IT \\ Arid Agriculture \\ University \\ Rawalpindi, Pakistan
}

\begin{abstract}
Performance enhancement of secondary communication, while adhereing to the interference constraint of the primary network in an underlay sepctrum sharing environemnt, is an active area of research. In this paper, we propose a Fuzzy Rule Based System (FRBS) assisted relay selection and transmit power allocation (RSTPA) technique that provides the secondary users the ability to coexist with the primary users in an energyconstrainted dual-hop Cognitive Radio Network. In this proposal, FRBS selects the optimal combination of relays aiming to maximize the signal-to-noise ratio (SNR) received at the destination, while guaranteeing that interference threshold of the primary network is not exceeded. The proposed scheme has been investigated for well-defined range of certain parameters. Simulation results prove that FRBS can accurately select the best combination of relays and maximize the SNR. Its performance in terms of end-to-end SNR has been compared with another scheme in the literature for multiple relay selection.
\end{abstract}

\section{Keywords}

Cognitive Radio Network, Underlay Spectrum Sharing, Cooperative Communication, Amplify-and-Forward, Fuzzy Rule Based System.

\section{INTRODUCTION}

Relaying has emerged as a promising spatial diversity technique in which multiple nodes collaborate with each other and relay the information from the source to the destination [1]. Such cooperative communication has gained much attention as it increases the diversity order of the signal, enables communication in the scenarios of deep fading, reduces power consumption and increases coverage area [2]. These factors make relay networks an attractive choice for Cognitive Radio Networks (CRNs) operating in an underlay spectrum sharing environment. In an underlay mode [3], secondary users (SUs) are allowed to coexist with the primary users (PUs), but at the cost of reduced transmit power in order to ensure the predefined interference threshold of the PU. This situation becomes even worst when line-of-sight (LOS) path between source-destination pair suffers from deep fading, thus making direct communication impossible. Fortunately, Relay assisted Cognitive Radio Networks (RCRNs) offer a potential solution to such problems. RCRNs are strongly motivated by two promising techniques, CR and user cooperation [4], and proved to be the best choice to provide minimum quality-of-service (QoS) requirements to the secondary networks in these scenarios. However, a CRN employing all the relays in the relay network may not be a feasible idea in an underlay environment, because it also increases the interference offered to the co-existing primary network. Fortunately, RCRNs incorporate a fascinating solution to such problems in the form of relay selection. Most commonly employed relaying strategies are Amplify-and-Forward (Non-Regenerative Relaying) and Decode-and-Forward (Regenerative Relaying) [5]. Amplify-and-Forward (AF) is the simplest relaying protocol in which a relay just scales the received message and forwards it to the destination.

Due to the above mentioned limitations of the underlay CRNs, relay selection and transmit power allocation to the SUs to maximize secondary system performance keeping in view the privilege of PUs is an active research area [6]. The optimal transmit power allocation requires information about power received from source to each relay, interference threshold of the primary network, channel state information (CSI) of source-relay, relay-destination, relay-PU links and the noise power received at each node in the network. There have been considerable research contributions on the interference management in this scenario. [7,8,9] consider two-hop underlay CRN with AF relays and present single and multiple relay selection schemes to maximize end-to-end SNR while satisfying the interference constraint. The highlighted contributions assume the absence of LOS path between the source-destination pair. Furthermore, the interference from primary transmitter towards the secondary network is also ignored, however only [8] considers the interference from source to PU.

Application of fuzzy systems in wireless communication is quite a recent area of -research. In [10], the authors proposed FRBS for adaptive communication in orthogonal frequency division multiplexing (OFDM) systems. In this proposal, FRBS was used to adapt optimum modulation code pairs (MCPs) for individual subcarriers depending upon the CSI at the subcarriers. Fuzzy Logic (FL) methodology has already been employed in CRs in the areas of spectrum sensing [11][13], interference management and power control [14]. Fuzzyrule-based power control mechanisms provide SUs the ability to dynamically adjust their transmit power in response to the changing network conditions. Few research contributions in the area of power management for spectrum sharing in CRNs are highlighted as follows. Le et. al designed a fuzzy power controller to enable secondary transmissions in the frequency band of the PU in [15]. [16] proposed a FL based transmit power controller to enhance secondary communication performance while considering the interference offered to the PU. In [17], Mustafa et. al considered the coexistence of primary and secondary networks, and for this scenario proposed FL based power control technique to reduce the bit error rate and ensuring the desired QoS requirements for the primary network. In [18], the authors proposed FL based power management strategy for secure spectrum sharing in CRNs. 
However, in energy-constrained RCRNs, no one has employed FL to perform multiple relay selection for the purpose of performance enhancement of secondary communication while satisfying the interference threshold of the primary network to the best of our knowledge.

In this paper, a CRN employing multiple relays between source destination pair is employed, while keeping in view all the above mentioned requirements. A novel idea of using FRBS for multiple relay selection and transmit power allocation scheme is proposed in order to select an optimal combination of relays which is able to maximize the SNR received at the destination while guaranteeing to satisfy the interference constraint. We also compare the performance of our proposed FRBS assisted multiple relay selection scheme with the greedy scheme of multiple relay selection presented in [19] and prove that the proposed scheme of relay selection chooses the optimum combination of relays and outsmart the greedy scheme in achieving high SNR.

The remaining paper is organized as follows. The system model is explained in section-II along with the mathematical formulation of the problem. The proposed fuzzy rule based multiple relay selection and transmit power allocation scheme is explained in section-III. Section-IV depicts the simulation results followed by section- $\mathrm{V}$ which concludes the whole paper.

\section{SYSTEM MODEL AND PROBLEM FORMULATION}

We consider CRN comprising a source-destination pair assisted by $M$ relays having $C R$ capabilities in their communication. The LOS path suffers from deep fading so the end-to-end communication between the source-destination pair is entirely dependent on the potential relay network. The entire relay network operates in an underlay spectrum sharing environment, thus simultaneous primary and secondary transmissions are allowed, but at the cost of reduced transmit power at the secondary network. The transmit power limitation is due to the intereference constraint imposed by the PU on the co-existing secondary network in order to keep the primary communication undisturbed, which in turn severely degrades secondary communication performance and limits the coverage area. The source is assumed to be far away from the PU, thus not interrupting primary communciation. The dual hop network under consideration is shown in Figure 1.

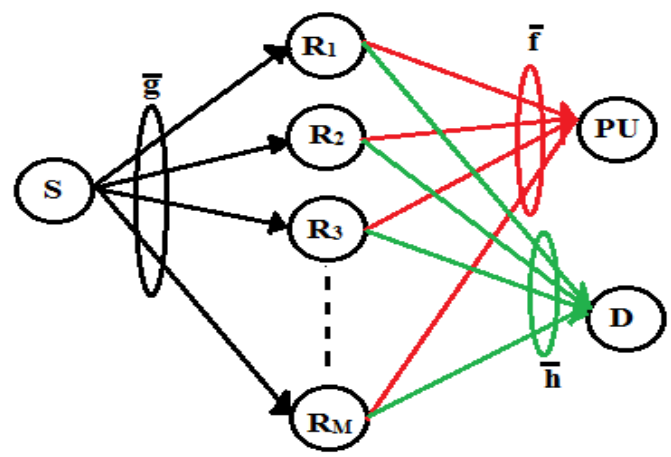

Fig 1: The System Model

In this Rayleigh flat fading scenario, let $\left[g_{m}\right]_{m=1}^{M},\left[h_{m}\right]_{m=1}^{M}$ and $\left[f_{m}\right]_{m=1}^{M}$ denote the Independent and Identically Distributed
(I.I.D.) $m^{\text {th }}$ channel coefficients between source-relay, relaydestination and relay-PU links. It is further assumed that perfect CSI is available at each relay.

The relays employ AF relaying and operate in half-duplex mode, thus, the end-to-end communication takes place in two time-slots. In the first time-slot, the source broadcasts the message which is received by the entire relay network. The signal received at the $m^{\text {th }}$ relay is given by,

$P_{m}=A_{m}^{2}\left(P_{S}\left|g_{m}\right|^{2}+N_{0}\right)$

where, $P_{S}$ is the transmit power of the source, $A_{m}$ denotes the amplification factor of the $m^{\text {th }}$ relay and $N_{0}$ is the Additive White Gaussian Noise (AWGN) term. The received message is amplified by each relay and forwarded to the destination in the second time-slot.

The end-to-end SNR received at the destination due to $m^{\text {th }}$ relay link is given by [20],

$$
\gamma_{m}=\frac{\gamma_{S R_{m}} \gamma_{R_{m} D}}{1+\gamma_{S R_{m}}+\gamma_{R_{m} D}}
$$

where,

$$
\gamma_{S R_{m}}=\frac{P_{S}\left|g_{m}\right|^{2}}{N_{0}} \text { and } \gamma_{R_{m} D}=\frac{P_{m}\left|h_{m}\right|^{2}}{N_{0}} \text { denote the }
$$

SNR of first and second hop, respectively.

Thus, the total end-to-end SNR due to the potential relay network is given as,

$$
\gamma_{D}=\sum_{m=1}^{M} \gamma_{m}=\sum_{m=1}^{M} \frac{\gamma_{S R_{m}} \gamma_{R_{m} D}}{1+\gamma_{S R_{m}}+\gamma_{R_{m} D}}
$$

As stated above, enabling the secondary communication while utilizing all relays might not be a viable idea in order to satisfy the interference constraint given by,

$$
I=\sum_{m=1}^{M} I_{m}=\sum_{m=1}^{M} P_{m}\left|f_{m}\right|^{2}<\zeta
$$

where, $\zeta$ denotes the interference threshold. This key requirement of the underlay spectrum sharing environment limits the transmit power of the relays which in turn reduces the SNR received at the destination. Thus, relay selection is performed to choose the best combination of relays which is able to maximize the received SNR keeping in view the privilege of the PUs. Therefore, FRBS is designed for multiple relay selection to choose the optimal combination of relays aiming to maximize secondary network performance in this scenario. The proposed FRBS will be explained in the next section. Forwarding the amplified message by the selected subset of relays takes place in the second time-slot. Thus, eq.(3) takes the form under multiple relay selection problem as,

$\gamma_{D}=\sum_{m \in \Gamma_{s}} \gamma_{m}=\sum_{m \in \Gamma_{s}} \frac{\gamma_{S R_{m}} \gamma_{R_{m} D}}{1+\gamma_{S R_{m}}+\gamma_{R_{m} D}}$ 
where $\Gamma_{s}$ denotes the subset of relays selected to forwarded the received message after amplification.

In literature, tight upper and lower bounds for $\gamma_{m}$ exist for a comprehensive performance analysis [21]. In terms of these bounds, $\quad \gamma_{m}$ is expressed as, $\gamma_{m}^{l b} \leq \gamma_{m} \leq \gamma_{m}^{u b}$, where, $\gamma_{m}^{l b} \leq \frac{1}{2} \min \left(\gamma_{S R_{m}}, \gamma_{R_{m} D}\right) \quad$ and $\quad \gamma_{m}^{u b} \leq \min \left(\gamma_{S R_{m}}, \gamma_{R_{m} D}\right)$ The bounds clearly indicate that $\gamma_{m}$ solely depends on $\gamma_{S R_{m}}$ when $\gamma_{R_{m} D}>\gamma_{S R_{m}}$. However, $\gamma_{m}$ can be enhanced for the case when $\gamma_{R_{m} D}<\gamma_{S R_{m}}$ to approach the upper bound. Thus, any attempt to improve $\gamma_{m}$ of those relays which exhibit $\gamma_{S R_{m}}<\gamma_{R_{m} D}$ will not result in any significant enhancement as $\gamma_{m}<\gamma_{S R_{m}}$ in this case.

Based on the above discussion, the multiple relay selection and power allocation problem can be mathematically expressed as,

$$
\begin{aligned}
& \max \left(\gamma_{2}=\sum_{m \in \Gamma_{s}} \gamma_{R_{m} D}\right) \\
& \text { s.t. } \\
& I=\sum_{m \in \Gamma_{s}} I_{m} \leq \zeta
\end{aligned}
$$

where, $\Gamma_{s}$ denotes the selected set of relays.

\section{FRBS ASSISTED RELAY SELECTION}

The FRBS assisted RSTPA scheme is explained in this section. The fuzzy logic system (FLS) is a two stage system as shown in Figure 2.

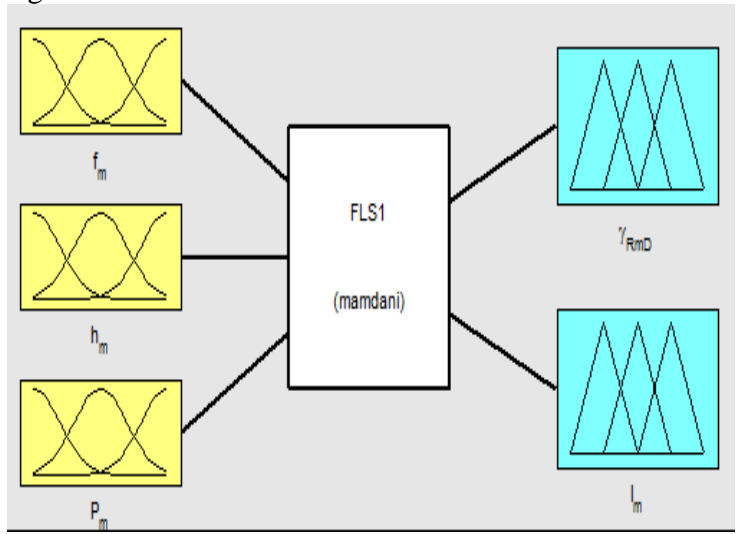

Fig 2(a): FLS 1

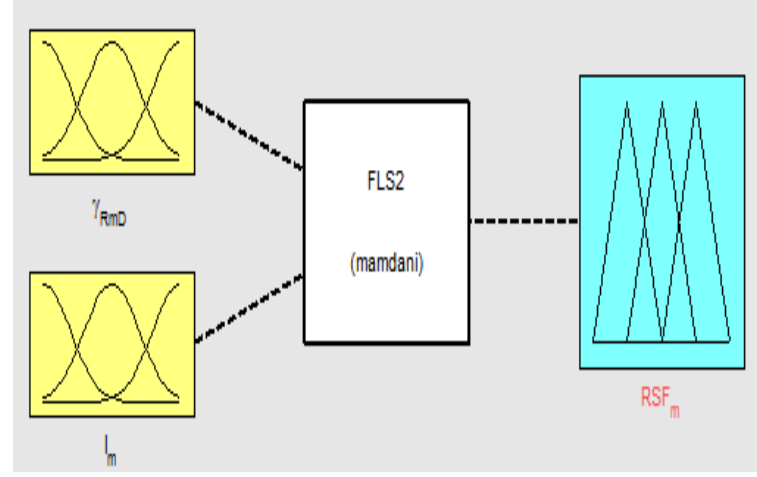

Fig 2(b): FLS 2

Fig 2: The proposed Fuzzy Rule based System As shown in the Figure 2, FLS 1 takes $P_{m}, h_{m}$ and $f_{m}$ corresponding to each $m^{\text {th }}$ relay as inputs and the fuzzy inference engine computes two outputs, one is $\gamma_{R_{m} D}$ achieved on the link from $m^{\text {th }}$ relay to destination, and the other is the interference $I_{m}$ offered to the PU at the $m^{\text {th }}$ relay link corresponding to each rule. The consequents of FLS 1 are then fed to FLS 2 shown in Figure 2(b) to determine the relay selection factor RSF for each $m^{\text {th }}$ relay. The membership functions (MFs) of the antecedents and consequents of FLS 1 and FLS 2 are given in Figure 3.

Referring to the MFs shown in figure 3, three fuzzy sets are used for the first input variable $f_{m}$, four fuzzy sets for the second input variable $h_{m}$, and six fuzzy sets for the third input variable $P_{m}$. Thus, there are $3 \times 4 \times 6=72$ "IF-THEN" rules for FLS 1 of the form,

$$
R^{l}: \text { IF } P_{1}^{l} \text { is } P_{6}, h_{1}^{l} \text { is } h_{4}, f_{1}^{l} \text { is } f_{3}
$$

\section{THEN}

$\gamma_{R_{1} D}^{l}$ is $S, I_{1}^{l}$ is $N S$

$$
l=1,2, \cdots, L
$$

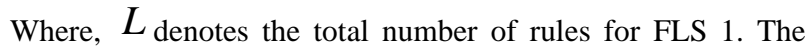
outputs $\gamma_{R_{m} D}$ and $I_{m}$ for each $m^{\text {th }}$ relay from FLS 1 are then fed to FLS 2 to determine the consequent Relay Selection Factor (RSF) of each $m^{\text {th }}$ relay. In FLS 2, there are $5 \times 5=25$ "IF-THEN" rules of the form,

$R^{k}: I F \gamma_{R_{1} D}^{k}$ is $S C S, I_{1}^{k}$ is $S$

\section{THEN}

$R S F_{1}^{k}$ is $S$

$$
k=1,2, \cdots \cdots, K
$$

where $K$ represents the total number of rules for FLS 2. The consequents of both fuzzy logic systems FLS1 and FLS 2 are 
divided into five levels: Not Selected (NS), Weak Consideration for Selection (WCS), Consider for Selection (CS), Strong Consideration for Selection (SCS) and Selected (S). Triangular MFs are used to represent the antecedent $f_{m}$, whereas, for all other cases of antecedents and consequents, trapezoidal MFs are chosen for minimum and maximum levels and triangular MFs are used to represent medium levels. Finally, the defuzzifier provides crisp value of RSF for each relay. Standard Center Average Defuzzifier is employed for this purpose. The standard Mamdani Inference Engine (MIE) is employed in both fuzzy logic systems to map each input combination to a particular output combination in FLS 1 and to a particular output in FLS 2. Also it uses MAX and MIN for all $\mathrm{s}$-norm and t-norm operators respectively.
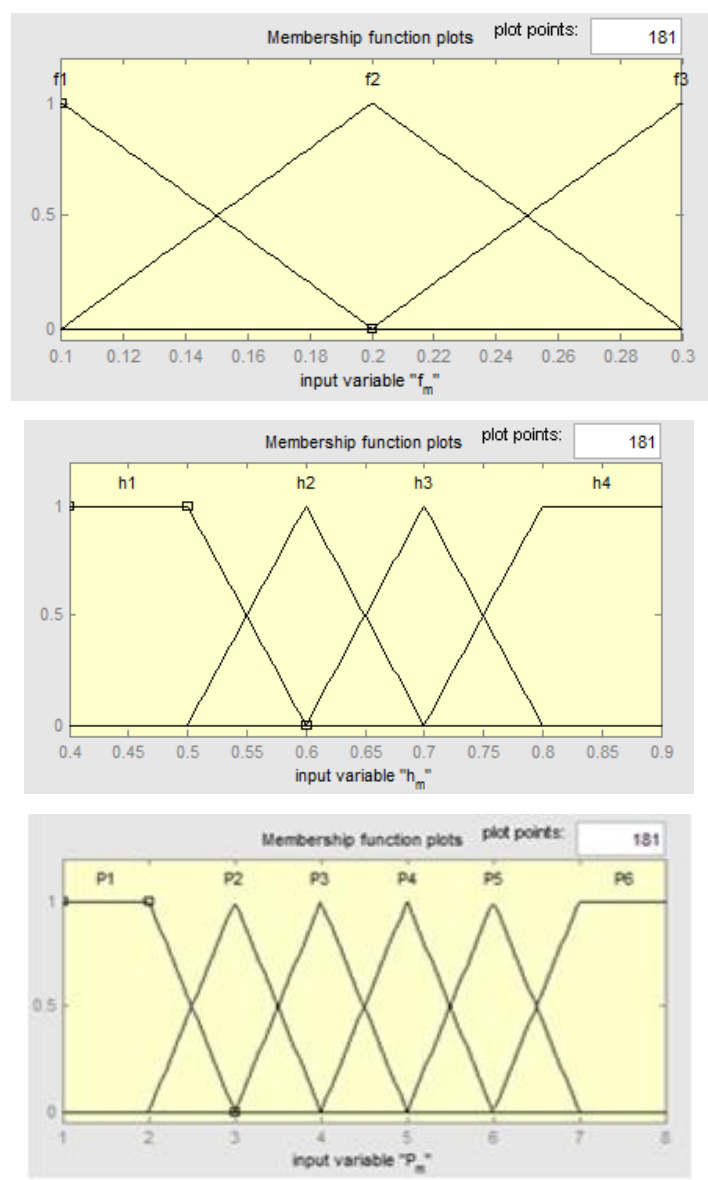
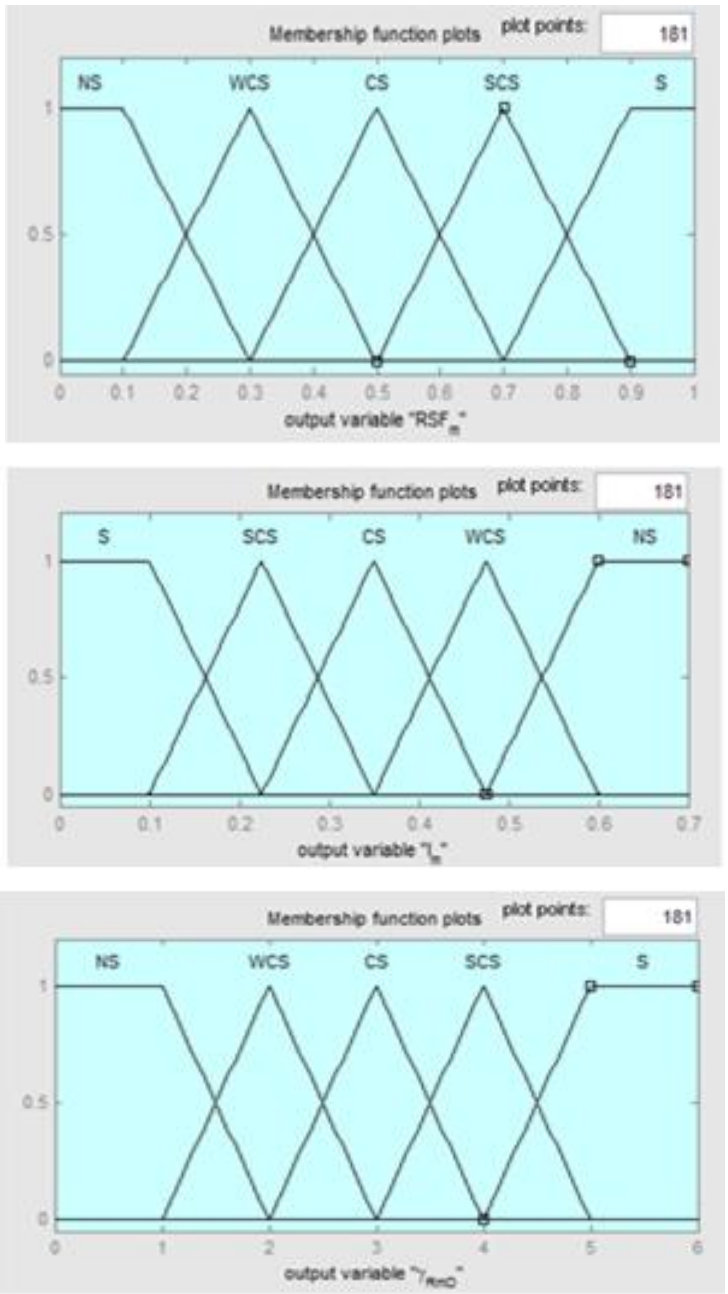

Fig 3: Membership Functions of Antecedents and Consequents

The flowchart of the proposed algorithm is shown in Figure 4. 


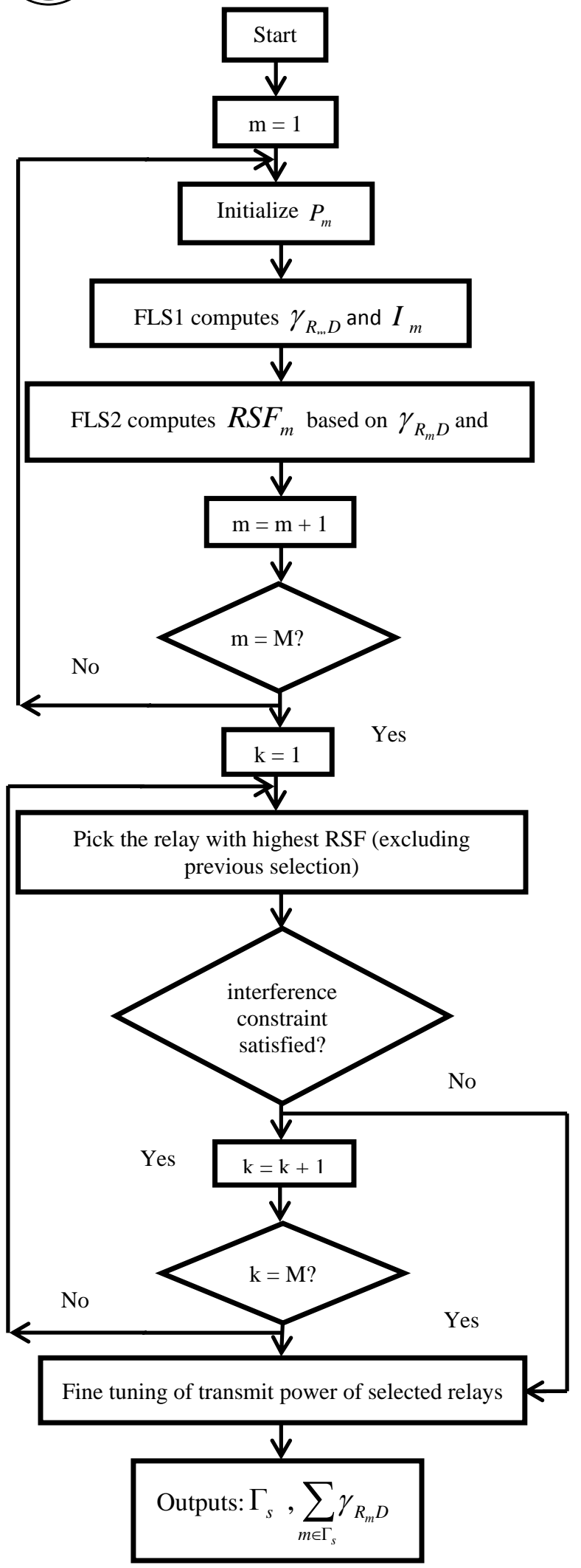

Fig 4: Flow Chart of Proposed Fuzzy Rule based Design

The algorithm works as follows: FLS 1 computes

$\gamma_{R_{m} D}$ and $I_{m}$ based on the CSI available at each relay, followed by FLS 2 which assigns RSF to each relay on the basis of consequents of FLS 1. Then the relays are picked up one by one in the descending order of RSF while ensuring to satisfy $\zeta$. Finally, the fine tuning of the transmit power of the relay with highest RSF is carried out aiming to maximize the SNR received at the destination. The relay with highest RSF is chosen for this purpose, because it exhibits the best channel condition towards the destination and at the same time it offers the minimum interference to the nearby PU.

\section{SIMULATION RESULTS}

Our proposed FRBS employs Mamdani Inference Engine (MIE) to select the optimal combination of relays and provides adjustment of transmit power of each selected relay. FRBS is designed in MATLAB 9.0 standard Fuzzy System Toolbox. Figure 5 shows the rule surface for FLS1 and FLS 2. The parameter settings for FRBS assisted relay selection algorithm are provided in Table 1.
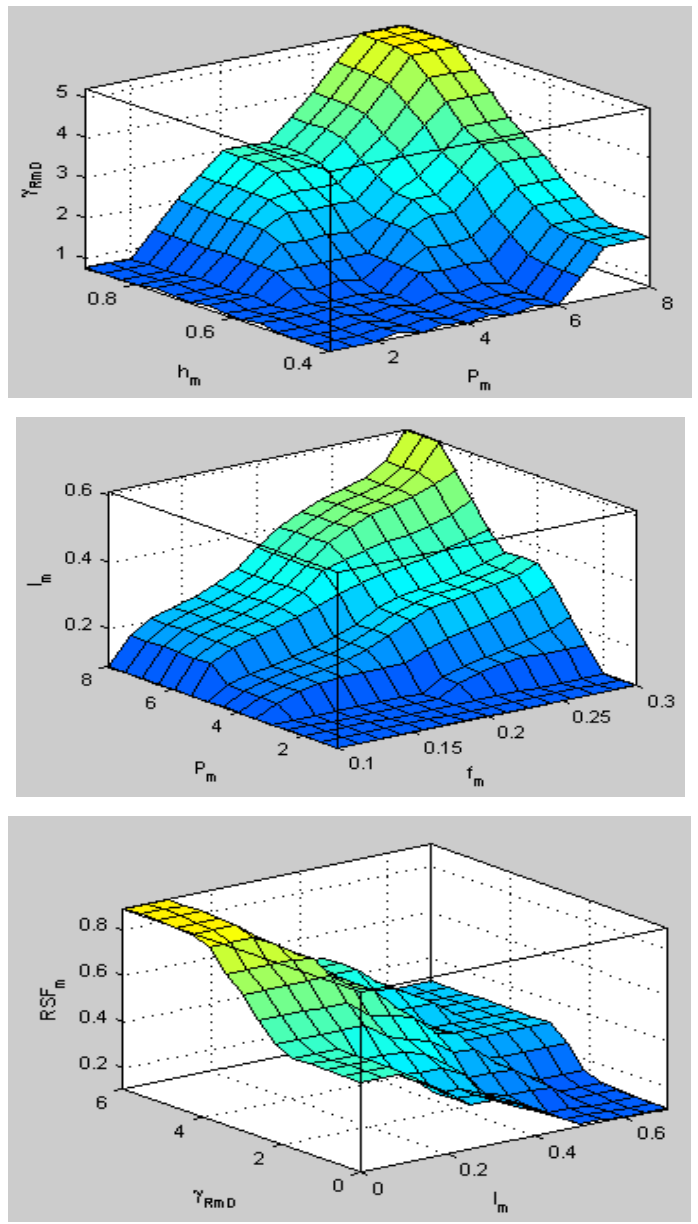

Fig 5: Rule Surface

Table 1: Parameter Settings

\begin{tabular}{|c|c|}
\hline Parameters & Values \\
\hline$g_{m}$ & $0.3-0.9$ \\
\hline$f_{m}$ & $0.1-0.3$ \\
\hline$h_{m}$ & $0.4-0.9$ \\
\hline$N_{0}$ & 1 \\
\hline
\end{tabular}

Figure (6) illustrates the performance of the proposed fuzzy 
rule based RSTPA scheme in terms of $\gamma_{2}$ given in eq. (6) achieved for different values of $\zeta P_{S}$ is set to 10 . We consider three different cases of total number of potential relays taken to be $M=\{5,10,15\}$. From Figure 6(a), it is observed that SNR increases significantly by relaxing $\zeta$ due to the fact that increasing $\zeta$ allows the relays to transmit at high power thus enhancing secondary network's performance. Furthermore, another strong observation is that, as the relay network grows, there is considerable improvement in the SNR, as large relay network adds more flexibility to choose those relays according to FRBS, which exhibit favorable channel conditions towards the destination and at the same time are not harmful for the primary communication. Figure 6(b) shows the corresponding number of selected relays for different cases considered in Figure 6(a). As observed from the figure, number of selected relays increases when $\zeta$ is relaxed and full cooperative diversity is achieved at very high values, in our case achieved for $\zeta \geq 10 \mathrm{~dB}$, whereas and on the other hand, the multiple relay selection problem reduces to single relay selection when interference threshold is held tight, i.e. $\zeta=-10 d B$ in our case. Thus, below this interference threshold level, secondary

communication will not be possible.

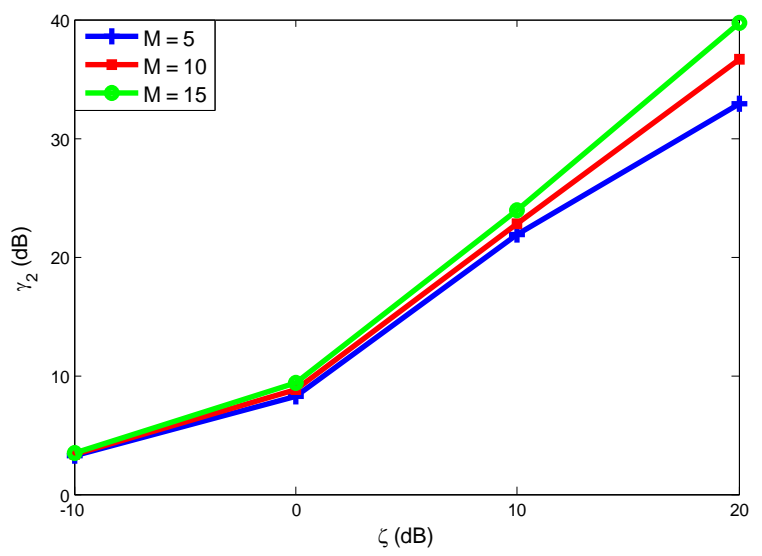

Fig 6(a)

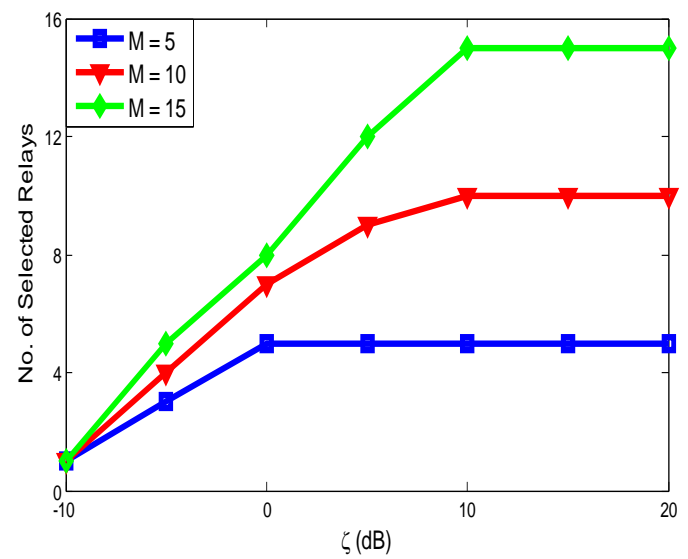

Fig 6(b)

Fig (6): (a) SNR performance of the proposed scheme, (b) Number of Corresponding Selected Relays
In Figure 7, the proposed FRBS is extended to evaluate $\gamma_{2}$ given in eq. (6) against different levels of $P_{S} . \zeta$ is set to 10 We consider the system's response for different number of potential relays $M=\{5,10,15\}$. The simulation results show the improvement in received SNR for low and medium levels of $P_{S}$, but SNR decreases at high transmit power levels. This is due to the reason that the higher is the power received at the relay network, the higher is the total interference offered by the relay network to the PU, which makes the relay selection problem difficult and reduces the number of selectedrelays, thus resulting in performance degradation. Thus, in an energy-constrained relay-assisted networks, where the interference threshold limits the transmit power of the relays, increasing the transmit power of the source does not play significant role in the communication performance enhancement.

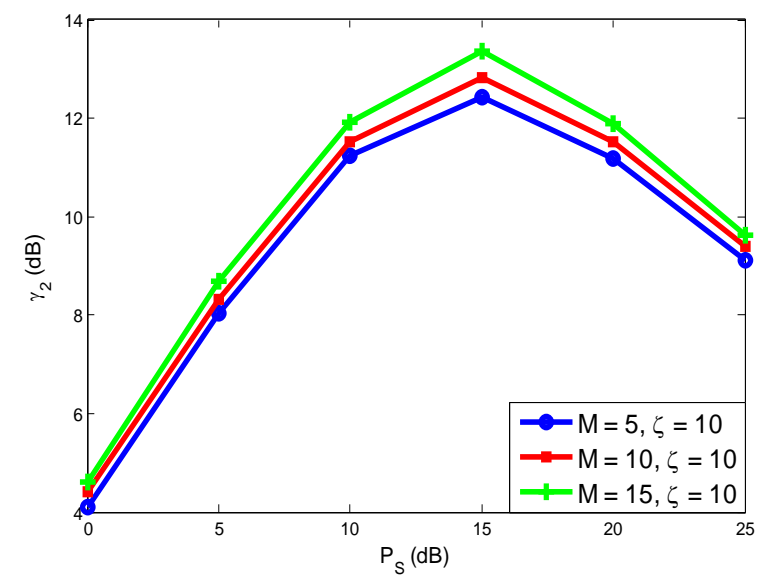

Fig 7: SNR Achieved by the proposed scheme for different levels of $P_{S}$

Figure 8 compares the performance of the proposed scheme with the greedy scheme [19] for multiple relay selection. The comparison has been carried out in terms of end-to-end SNR given in eq. (5). All parameter settings are done according to [19]. The simulation results prove that the proposed scheme is able to achieve high end-to-end SNR as compared to the greedy scheme. This improvement is achieved due to two strong factors. First, FRBS assisted RSTPA design checks every possible combination of relay-destination and relay-PU channel coefficients and picks up the best combination of relays to enhance secondary performance while operating in an underlay mode. Second, the flexibility in the transmit power allocation to the selected relays with the priority given to the relay with the highest RSF obtained from FRBS keeping in view the interference threshold. On the other hand, in the greedy scheme, either the relay does not participate in the communication or transmits at fixed power $P_{m}=P_{S}$, thus, there is no flexible transmit power allocation for the selected relays for enhancement of secondary communication. However, in high power areas, the SNR decreases in both cases due to the same reason mentioned above. 


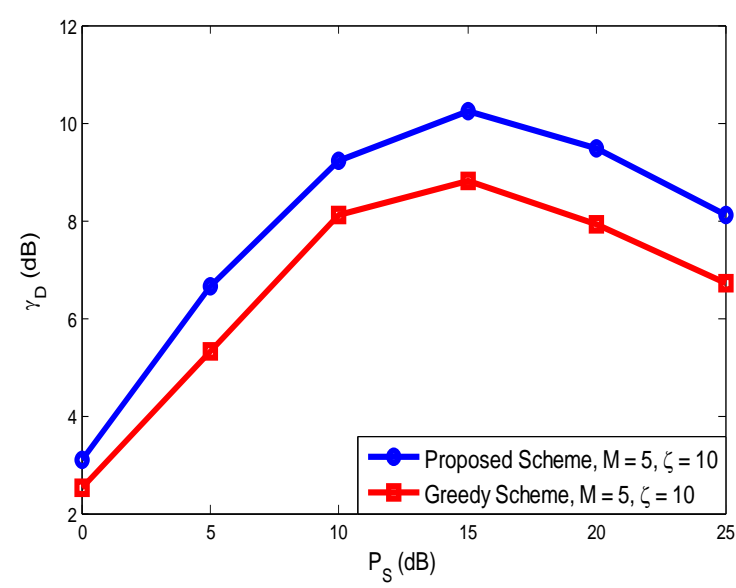

Fig 8: Performance Comparison of the Proposed Scheme and the Greedy Scheme

\section{CONCLUSION}

In this work, we propose a novel fuzzy rule based relay selection and transmit power allocation scheme to enable the secondary communication in the frequency band of primary users. Once the CSI based knowledge is pumped into the FRBS along with the power received at each relay, it provides the optimum relay selection factor. RSF obtained from the FRBS, is used as a priority factor that highlights the relays which exhibit the ability to enhance secondary system performance and are less harmful to the primary users. We also proved that the proposed fuzzy logic based relay selection scheme outsmarts an existing scheme in literature for multiple relay selection. In future work, we aim to propose a more sophisticated FRBS assisted multiple relay selection techniques considering different parameters with an objective to maximize the secondary system performance in an underlay spectrum sharing environment.

\section{REFERENCES}

[1] Nikjah, R., Beaulieu, N.C.. 2009. Exact Capacity Analysis of Rate Adaptive Power Non adaptive Multibranch Multihop Decode-and-Forward Relaying Networks. GLOBECOM IEEE. 1-8.

[2] Laneman, J.N.; Wornell, G.W.. 2000. Energy Efficient Antenna Sharing and Relaying for Wireless Networks. WCNC IEEE. 7-12.

[3] Im S., Kim W, Kang Y., Lee H. 2010. Joint Power and Admission Control for Underlay Spectrum Sharing in Cognitive Radio Networks. ATC IEEE. 56-6.

[4] Sultan K., Qureshi I.M., Zubair M. 2012. SNR Maximization through Relay Selection and Power Allocation for Non-Regenerative Cognitive Radio Networks. INMIC IEEE. 361-364.

[5] Gomaa A., Mokhtar M., Al-Dhahir N. 2012. Amplifyand-Forward Relaying under I/Q Imbalance. GLOBECOM IEEE. 4671-4676.

[6] Sun C., Letaief K. B. 2008. User Cooperation in Heterogeneous Cognitive Radio Networks with Interference Reduction. ICC IEEE, 3193-3197.
[7] M. Naeem, D.C. Lee, U. Pareek, 2010. An Efficient Multiple Relay Selection Scheme for Cognitive Radio Systems", Proceedings of IEEE International Conference on Communications, 1-5.

[8] M.A. Manna, G. CHEN, J.A. CHAMBERS. 2014. Outage Probability Analysis of Cognitive Relay Network with Four Relay Selection and End-To-End Performance with Modified Quasi-Orthogonal Space-Time Coding", IET Commun., vol. 8, no. 2, 233-241.

[9] G. Chen, O. Alnatouh, J. Chambers., 2013. Outage Probability Analysis for A Cognitive Amplify-AndForward Relay Network With Single and Multi-Relay Selection", IET Commun., vol. 7, no. 17, 1974-1981.

[10] Atta-ur-Rahman, Qureshi I.M., Malik A.N. 2012. A Fuzzy Rule Base Assisted Adaptive Coding and Modulation Scheme for OFDM Systems. J. Basic Appl. Sci. Res. Vol. 2(5), 4843-4853.

[11] Liu W., Lv T., Gao L., Wang W., Liu B. 2009. A Novel Cooperative Spectrum Sensing Scheme Based on Fuzzy Integral Theory in Cognitive Radio Networks. WiCom IEEE. 1-4.

[12] Zhang H., Wang X. 2011. A Fuzzy Decision Scheme for Cooperative Spectrum Sensing in Cognitive Radio. VTC IEEE. 1-4.

[13] Ejaz W., ul Hasan, N., Aslam, S., Kim H. S. 2011. Fuzzy Logic Based Spectrum Sensing for Cognitive Radio Networks", NGMAST IEEE. 185-189.

[14] Aryal S. R., Dhungana H., Paudyal K. 2012. Novel Approach for Interference Management in Cognitive Radio. AH-ICI IEEE. 1-5.

[15] Le H.S.T., Liang $\quad 2007$. An Efficient Power Control Scheme for Cognitive Radios. WCNC IEEE. 2559-2563.

[16] Tabakovic Z., Grgic S., Grgic M. 2009. Fuzzy Logic Power Control in Cognitive Radio. IWSSIP IEEE. 1-5.

[17] Mustafa, W. , Yu J.S., Rakus-Andersson, E. , Mohammed, A., Kulesza, W.J. 2010. Fuzzy-based Opportunistic Power Control Strategy in Cognitive Radio Networks. ISABEL IEEE. 1-9.

[18] Dey A., Biswas S., Panda S. 2011. A New Fuzzy Rule Based Power Management Scheme for Spectrum Sharing in Cognitive Radio. ICCIA IEEE. 1-4.

[19] Xu J., Zhang H., Yuan D., Jin Q., Wang C. 2011. Novel Multiple Relay Selection Schemes in Two-Hop Cognitive Relay Networks. CMC IEEE. 307-310.

[20] Hui H., Zhu S. , Lv G. 2010. Relay Selection for Lifetime Extension in Amplify-and-Forward Cooperative Networks. ICC IEEE. 1-5.

[21] Amarasuriya G., Ardakani M., Tellambura C. 2010. Output-Threshold Multiple-Relay-Selection Scheme for Cooperative Wireless Networks. IEEE Transactions on Vehicular Technology. Vol. 59(6). 3091-3097. 